\title{
Comparison between magnetic coplanarity and MVA methods in determining the normal of Venusian bow shock
}

\author{
SHAN LiCan, LU QuanMing ${ }^{*}$, ZHANG TieLong, GAO XinLiang, HUANG Can, \\ SU YanQing \& WANG Shui
}

CAS Key Laboratory of Basic Plasma Physics, Department of Geophysics and Planetary Science, University of Science and Technology of China, Hefei 230026, China

Received August 21, 2012; accepted September 28, 2012; published online February 1, 2013

\begin{abstract}
With the measurements of magnetic field of Venus Express (VEX), magnetic coplanarity and minimum variance analysis (MVA) methods are analyzed and their validity is tested to determine the normal of Venusian bow shocks. It is found that MVA method is the better than magnetic coplanarity, and 95\% shock crossings can be accurately determined by the method. However, the occurrence of the shock normal which is not determined accurately by magnetic coplanarity increases with the decrease of the solar zenith angle (SZA). At the same time, compared with quasi-parallel shocks, there is more occurrence of the shock normal which cannot be determined accurately by magnetic coplanarity for quasi-perpendicular shocks.
\end{abstract}

MVA, magnetic coplanarity, Venusian bow shock, shock normal

Citation: Shan L C, Lu Q M, Zhang T L, et al. Comparison between magnetic coplanarity and MVA methods in determining the normal of Venusian bow shock. Chin Sci Bull, 2013, 58: 2469-2472, doi: 10.1007/s11434-013-5675-8

The physics of collisionless shock is a very broad topic in space and astrophysical plasmas [1-3]. A shock plays an important role in converting upstream bulk flow energy into downstream thermal energy, across which the plasma speed decreases from super-magnetosonic in the upstream to sub-magnetosonic in the downstream. A controlling factor, which determines the characteristics of a collisionless shock, is the magnetic geometry of the shock, usually described by the angle $\theta_{B n}$ between the shock normal and the upstream magnetic field direction. Quasi-parallel $\left(\theta_{B n}<45^{\circ}\right)$ and quasiperpendicular shocks $\left(\theta_{B n}>45^{\circ}\right)$ have very different internal structure and thermalization processes. A quasi-perpendicular shock has an ion cyclotron scale foot and an overshoot after ramp [4], while low-frequency, large amplitude turbulence is ubiquitous prior to and after quasi-parallel shock. The dissipation mechanism from upstream bulk flow energy into downstream thermal energy is also different between a quasi-parallel and quasi-perpendicular shock. Therefore, determining the normal direction of a shock is the premise

*Corresponding author (email: qmlu@ ustc.edu.cn) to study the characteristics of a shock.

A planet bow shock is generated by the interaction between the tenuous, high speed solar wind and the magnetosphere, ionosphere and atmosphere of the planet. In addition, the heated ions created by charge exchange or photoionization of cold neutral atoms, escaping from planetary atmosphere, also play an important role in a planet bow shock [5,6], for example in Venusian bow shock. There are two analysis methods to determine the normal of a shock by single spacecraft observation, i.e. magnetic coplanarity [7-9] and minimum variance analysis (MVA) [10-12]. In this paper, with the magnetic field data obtained by Venus Express (VEX) spacecraft, we compare magnetic coplanarity and MVA methods in determining the normal of Venusian bow shock.

\section{Magnetic coplanarity and MVA methods}

Both magnetic coplanarity and MVA methods are based on a formula of $\nabla \cdot \mathbf{B}=0$. The normal components of the 
magnetic field in the upstream and downstream of a shock are equal. In addition, the two methods with single satellite observations rely on the assumption that the magnetic field in the upstream and downstream is stationary.

Magnetic coplanarity further assumes that the magnetic field in the upstream, downstream and the shock normal are in the same plane. The magnetic field in the upstream and downstream satisfies Rankine-Hugoniot relations. Figure 1 shows the schematic of magnetic coplanarity, and the shadow represents the shock layer. The shock normal can be determined by the following equation $[8,9]$ :

$$
\boldsymbol{n}= \pm \frac{\left(\mathbf{B}_{\mathrm{d}}-\mathbf{B}_{\mathrm{u}}\right) \times\left(\mathbf{B}_{\mathrm{d}} \times \mathbf{B}_{\mathrm{u}}\right)}{\left|\left(\mathbf{B}_{\mathrm{d}}-\mathbf{B}_{\mathrm{u}}\right) \times\left(\mathbf{B}_{\mathrm{d}} \times \mathbf{B}_{\mathrm{u}}\right)\right|}
$$

where $\mathbf{B}_{\mathrm{u}}, \mathbf{B}_{\mathrm{d}}$ are the average magnetic field in the upstream and downstream of the shock, respectively. The interval to calculate the average magnetic field is chosen to be $2 \mathrm{~min}$ in this paper, and it takes the satellite 6-9 min from the shock to the magnetopause. The sign of $\boldsymbol{n}$ is arbitrary and the outward normal to the bow shock points upstream.

In order to determine the normal of the magnetopause current layer, Sonnerup and Cahill [10] developed MVA method. Then, Siscoe et al. [11] utilized this method to determine the normal of interplanetary current sheet. Recently, this method has been widely applied to determine the normal of the magnetopause, current sheet, shock and wave vector [13-18]. On the basis of $\nabla \cdot \mathbf{B}=0$, the normal component of the magnetic field, during shock crossing, has minimum variance [10],

$$
\sigma^{2}=\frac{1}{K} \sum_{k=1}^{K}\left|\left(\mathbf{B}^{(k)}-\langle\mathbf{B}\rangle\right) \cdot n\right|^{2},
$$

where $K$ is the number of the magnetic field measurements used in the calculation, $\langle\mathbf{B}\rangle$ is the average of those measurements. In order to calculate $n$ conveniently, eq. (2) can

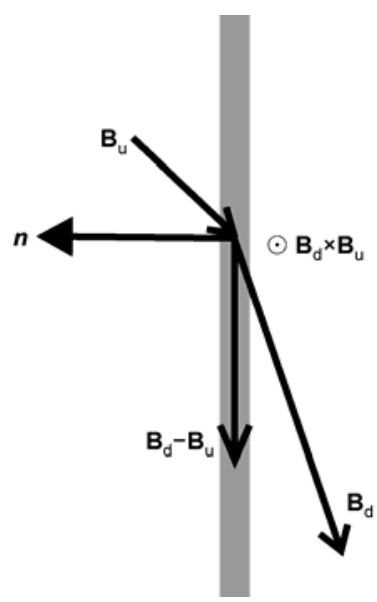

Figure 1 The schematic illustration of magnetic coplanarity. The shade represents shock layer. $\mathbf{B}_{\mathrm{u}}, \mathbf{B}_{\mathrm{d}}$ are the average magnetic field in the upstream and downstream of the shock, respectively. be written in magnetic variance matrix form,

$$
\left\{\begin{array}{l}
\mathbf{M} \cdot \boldsymbol{n}=\lambda n, \\
\mathbf{M}=\langle\mathbf{B B}\rangle-\langle\mathbf{B}\rangle\langle\mathbf{B}\rangle .
\end{array}\right.
$$

Here, $\lambda$ values are the eigenvalues of matrix equation. The smallest eigenvalue of $\lambda$ corresponds to the shock normal $\boldsymbol{n}$.

\section{The normal of Venusian bow shock}

Due to the lack of a global intrinsic planetary magnetic field, the super-magnetosonic solar wind interacts directly with the Venusian ionosphere, which forms an induced magnetosphere. The periapsis of VEX orbit is about $300 \mathrm{~km}$ with periapsis latitude at $78^{\circ} \mathrm{N}$, and the period of one VEX orbit is about $24 \mathrm{~h}$. Figure 2 displays one sample bow shock crossing observed by VEX from 06:06:00 to 06:16:00 UT on 20 November, 2006. The magnetic field data have $1 \mathrm{~s}$ time resolution [19], which are shown in Venus Solar Orbital (VSO, resembling GSE coordinates of the Earth) coordinates. In the VSO coordinates, the $x$ and $z$ axes point sunward and northward, respectively, while the $y$ axis completes the right-handed set. In the figure, the highlighted regions represent the magnetic field profiles in the upstream and downstream, respectively. In order to test the validity of magnetic coplanarity and MVA methods, a Venusian bow

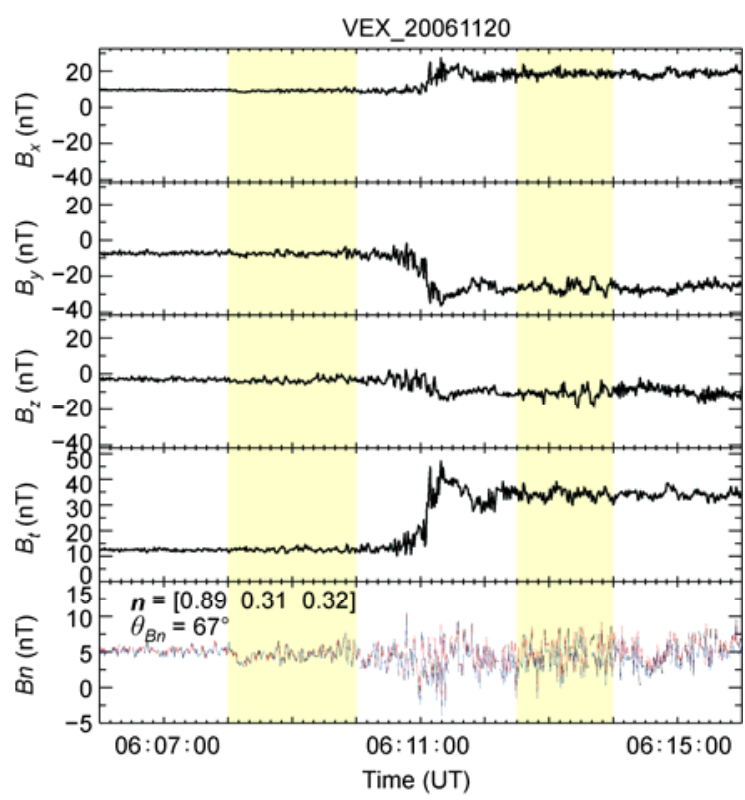

Figure 2 The profiles of $B_{x}, B_{y}, B_{z}, B_{t}=\sqrt{B_{x}^{2}+B_{y}^{2}+B_{z}^{2}}$ and $B n=\mathbf{B} \cdot \boldsymbol{n}$ observed by VEX between 06:06:00 and 06:16:00 UT on 20 November, 2006. The highlighted regions represent the upstream and downstream magnetic field profiles respectively. The shock normal $\boldsymbol{n}$ and $\theta_{B n}$ shown in the lowest panel are calculated based on MVA method, and dashed lines show the normal components of magnetic field determined by magnetic coplanarity (black), MVA (red) and Venusian bow shock model (blue), respectively. 
shock model [20] are also used in the paper. The normal components of the magnetic field determined by the three methods are also shown in the bottom panel. We can find that the three methods obtain consistent results in this crossing. It is a quasi-perpendicular shock.

We utilize totally 318 shock crossings based on VEX spacecraft observations from May of 2006 to August of 2011 to test the validity of magnetic coplanarity and MVA methods. Three different $\theta_{B n}$ can be obtained with the three different methods: magnetic coplanarity, MVA and the Venusian bow shock model. Only if when at least two methods get consistent results (the difference of $\theta_{B n}$ is smaller than $10^{\circ}$ ), we think that the obtained value can be considered as a reasonable value, while the other is unacceptable.

Figure 3 plots the occurrence percentage of the shock normal that is not determined accurately at different SZA ranges. It is noted that the occurrence percentage is calculated by normalizing the occurrence number to the total number (318 cases). MVA method is the best choice to determine the normal of Venusian bow shock, and about $95 \%$ cases can be correctly determined by MVA method. The occurrence percentage of the shock normal which cannot accurately determined by the Venusian bow shock model increases at large SZA, because the shock begins to oscillate when the SZA is large [20]. Magnetic coplanarity is not suitable for the cases at small SZA. The reason may be due to the smaller distance between the bow shock and ionosphere at small SZA, where the induced magnetic field disturbance of the magnetic field from the ionosphere is large.

Figure 4 plots the occurrence percentage of the shock normal which cannot be accurately determined at different $\theta_{B n}$, i.e. $0^{\circ}<\theta_{B n} \leqslant 30^{\circ}, 30^{\circ}<\theta_{B n} \leqslant 60^{\circ}$ and $60^{\circ}<\theta_{B n} \leqslant 90^{\circ}$. Compared with quasi-parallel shocks (especially at $0^{\circ}<\theta_{B n} \leqslant 30^{\circ}$ condition), the occurrence percentage of the shock normal which cannot be accurately determined by magnetic coplanarity is larger for quasi-perpendicular shocks.

In addition, 161 shock crossings of the Earth observed by

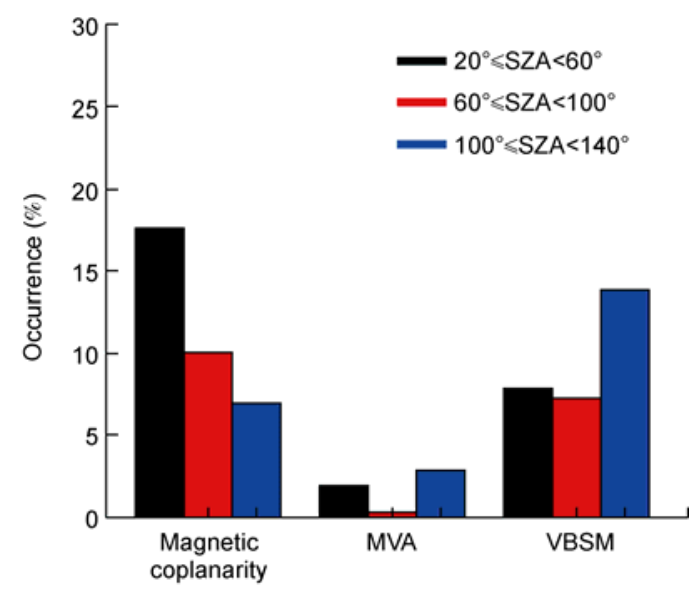

Figure 3 Histograms of occurrence percentage of the shock normal which is not determined accurately by magnetic coplanarity, MVA method, or the Venusian bow shock model (VBSM).

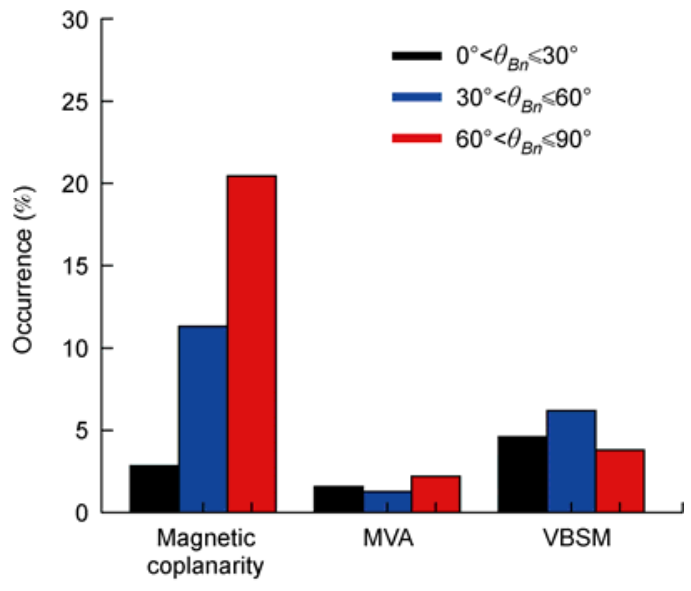

Figure 4 Histograms of percentage occurrence of the shock normal which cannot be accurately determined by magnetic coplanarity, MVA method, or the Venusian bow shock model (VBSM).

FGM of Cluster II are also analyzed to compare them with the Venusian bow shock. Here, the Earth's bow shock model of Peredo et al. [21] is used instead of the Venusian bow shock model [20]. Figure 5 plots the occurrence percentage of the shock normal which cannot be accurately determined at different $\theta_{B n}$. Because of few cases at $\theta_{B n} \leqslant 40^{\circ}$, only those shocks at $40^{\circ}<\theta_{B n} \leqslant 65^{\circ}$ and $65^{\circ}<\theta_{B n} \leqslant 90^{\circ}$ conditions are studied. It is also found MVA method is better than magnetic coplanarity. Besides, compared with the shocks at $40^{\circ}<\theta_{B n} \leqslant 65^{\circ}$, the occurrence percentage of the shock normal which cannot be accurately determined by magnetic coplanarity is larger at $65^{\circ}<\theta_{B n} \leqslant 90^{\circ}$.

\section{Conclusions}

In the present paper, 318 Venusian bow shock crossings observed by VEX spacecraft from May of 2006 to August of 2011 are analyzed to test the validity of magnetic copla-

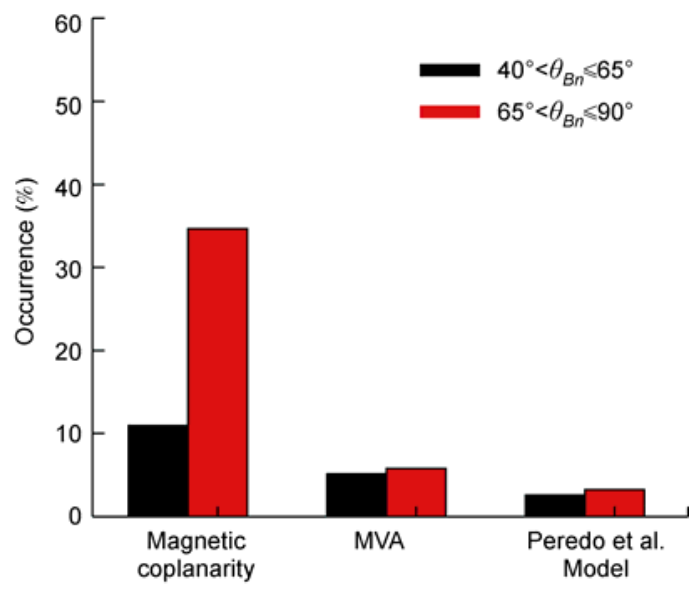

Figure 5 Histograms of occurrence percentage of the shock normal which is not determined accurately by magnetic coplanarity, MVA method, or the Earth's bow shock model. 
narity and MVA methods. The conclusions can be summarized as follows:

(1) MVA is the optimal method to determine the shock normal, and about 95\% Venusian shock crossings can be accurately determined. In general, there are large amplitude fluctuations when MVA method fails.

(2) The occurrence percentage of the shock normal which cannot be accurately determined by magnetic coplanarity increases when the SZA decreases.

(3) Due to the oscillation of shock at large SZA, the occurrence percentage of the shock normal which cannot be accurately by the Venusian bow shock model becomes large when the SZA is more than $100^{\circ}$.

(4) There is more occurrence percentage of the shock normal which cannot be determined accurately by magnetic coplanarity in quasi-perpendicular shocks (especially at $\left.\theta_{B n}>60^{\circ}\right)$.

(5) Conclusions (1) and (4) are also valid for the Earth's bow shocks.

In general, the shock normal may not be accurately determined by MVA method. when the ratio of the smallest eigenvalue to the intermediate eigenvalue is larger than $1 / 3$. In the present paper, among the cases whose shock normal is not considered to be accurately determined by MVA method, there are about $75 \%$ cases where the ratio is larger than $1 / 3$. However, among the cases whose shock normal is considered to be accurately determined by MVA method, there are only about $31 \%$ cases where the ratio is larger than $1 / 3$. Therefore, the ratio is a crucial parameter to determine the validity of MVA method [10].

This work was supported by Ocean Public Welfare Scientific Research Project, State Oceanic Administration People's Republic of China (201005017), the National Natural Science Foundation of China (40931053, 41174124, 41274144 and 41121003), and the Fundamental Research Funds for the Central Universities (WK2080000010). The authors thank the FGM team and the ESA Cluster Active Archive.

1 Walker S N, Balikhin M A, Nozdrachev M N. Ramp nonstationarity and the generation of whistler waves upstream of a strong quasiperpendicular shock. Geophys Res Lett, 1999, 26: 1357-1360

2 Chapman S C, Lee R E, Dendy R O. Perpendicular shock reformation and ion acceleration. Space Sci Rev, 2005, 121: 5-19
3 Yang Z W, Lembege B, Lu Q M. Acceleration of heavy ions by perpendicular collisionless shocks: Impact of the shock front nonstationarity. J Geophys Res, 2011, 116: A10202

4 Baumjohann W, Treumann R A. Basic Space Plasma Physics. London: Imperial College Press, 1997

5 Liewer P C, Goldstein B E, Omidi N. Hybrid simulations of the effects of interstellar pickup hydrogen on the solar-wind termination shock. J Geophys Res, 1993, 98: 15211-15220

6 Zank G P, Pauls H L. Shock propagation in the outer heliosphere 1. Pickup ions and gasdynamics. J Geophys Res, 1997, 102: 7037-7049

7 Colburn D S, Sonett C P. Discontinuities in the solar wind. Space Sci Rev, 1966, 5: 439-506

8 Lepping R P, Argentiero P D. Single spacecraft method of estimating shock normals. J Geophys Res, 1971, 76: 4349-4359

9 Abraham-Shrauner B. Determination of magnetohydrodynamic shock normals. J Geophys Res, 1972, 77: 736-739

10 Sonnerup B U, Cahill L J. Magnetopause structure and attitude from explorer 12 observations. J Geophys Res, 1967, 72: 171-183

11 Siscoe G L, Davis L Jr, Coleman P J Jr, et al. Power spectra and discontinuities of the interplanetary magnetic field: Mariner 4. J Geophys Res, 1968, 73: 61-82

12 Kawano H, Higuchi T. A generalization of the minimum variance analysis method. Ann Geophys, 1996, 14: 1019-1024

13 Lefebvre B, Seki Y, Schwartz S J, et al. Reformation of an oblique shock observed by Cluster. J Geophys Res, 2009, 114: A11107

14 Wilson L B, Cattell C A, Kellogg P J, et al. Low-frequency whistler waves and shocklets observed at quasi-perpendicular interplanetary shocks. J Geophys Res, 2009, 114: A10106

15 Zhang H, Zong Q G, Sibeck D G, et al. Dynamic motion of the bow shock and the magnetopause observed by THEMIS spacecraft. J Geophys Res, 2009, 114: A00C12

16 Du J, Zhang T L, Baumjohann W, et al. Statistical study of lowfrequency magnetic field fluctuations near Venus under the different interplanetary magnetic field orientations. J Geophys Res, 2010, 115: A12251

17 Cutler J C, Dougherty M K, Lucek E, et al. Evidence of surface wave on the dusk flank of Saturn's magnetopause possibly caused by the Kelvin-Helmholtz instability. J Geophys Res, 2011, 116: A10220

18 Dimmock A P, Walker S N, Zhang T L, et al. Spatial scales of the magnetic ramp at the Venusian bow shock. Ann Geophys, 2011, 29: 2081-2088

19 Zhang T L, Baumjohann W, Delva M, et al. Magnetic field investigation of the Venus plasma environment: Expected new results from Venus Express. Planet Space Sci, 2006, 54: 1336-1343

20 Zhang T L, Delva M, Baumjohann W, et al. Initial Venus Express magnetic field observations of the Venus bow shock location at solar minimum. Planet Space Sci, 2008, 56: 785-789

21 Peredo M, Slavin J A, Mazur E, et al. Three-dimensional position and shape of the bow shock and their variation with Alfvenic, sonic and magnetosonic Mach numbers and interplanetary magnetic field orientation. J Geophys Res, 1995, 100: 7907-7916

Open Access This article is distributed under the terms of the Creative Commons Attribution License which permits any use, distribution, and reproduction in any medium, provided the original author(s) and source are credited. 